\title{
On the Generality of Orthogonal Projections and e-Projections in Banach Algebras
}

\author{
Mohammad Sadegh Asgari, Seyedeh Sara Karimizad, Hamidreza Rahimi \\ Department of Mathematics, Faculty of Science, Islamic Azad University, Central Tehran Branch, Tehran, Iran \\ Email: moh.asgari@iauctb.ac.ir, s_karimizad@yahoo.com,rahimi@iauctb.ac.ir
}

Received March 2, 2012; revised April 18, 2012; accepted April 28, 2012

\begin{abstract}
In this paper we develop the orthogonal projections and e-projections in Banach algebras. We prove some necessary and sufficient conditions for them and their spectrums. We also show that the sum of two generalized orthogonal projections $u$ and $v$ is a generalized orthogonal projection if, $u v=v u=0$. Our results generalize the results obtained for bounded linear operators on Hilbert spaces.
\end{abstract}

Keywords: Generalized Orthogonal Projection; Orthogonal Projection; Generalized e-Projection; e-Projection

\section{Introduction}

Orthogonal projections on Hilbert spaces play important roles in many applications in mathematics, science and engineering including signal and image processing, integral equations and many other areas. In this article we introduce generalized orthogonal projections, generalized e-projections in Banach algebras and we show that they share many useful properties with their corresponding notions in $C^{*}$-algebras. For more information we refer to the articles by Berkson [1], Schmoeger [2], Du et al. [3], Grob et al. [4] and Lebtahi et al. [5].

The paper is organized as follows: Section 1, contains a few elementary definitions and results from Banach algebras theory. In this section we introduce the concepts of numerical range and the spectrum and the spectral radius of an element and investigate their properties. In Section 2, we introduce the generalized orthogonal projections, generalized e-projections in Banach algebras and we study some necessary and sufficient conditions for them and their spectrums.

Throughout this paper, $\mathcal{A}$ will denote a complex unital Banach algebras (with unit 1) and $\mathcal{A}^{\prime}$ denote the dual space of $\mathcal{A}$. For $x \in \mathcal{A},\|x\|=1$, define the support set at $x$

$$
D(\mathcal{A}, x)=\left\{\varphi \in \mathcal{A}^{\prime}:\langle x, \varphi\rangle=1=\|\varphi\|\right\}
$$

Then for all $a \in \mathcal{A}$ define the sets

$$
V(\mathcal{A}, a, x)=\{\langle a x, \varphi\rangle: \varphi \in D(\mathcal{A}, x)\}
$$

and their union, the numerical range of $a$

$$
V(\mathcal{A}, a)=\bigcup_{x \in \mathcal{A},\|x\|=1} V(\mathcal{A}, a, x)
$$

We also denote the spectrum and the spectral radius of $a$ by $\sigma(a)$ and $r(a)$ respectively.

Lemma 1.1. [6]. Let $a, b \in \mathcal{A}, \lambda \in \mathbb{C}$ then

1) $V(\mathcal{A}, a)$ is a compact convex subset of $\mathbb{C}$.

2) $V(\mathcal{A}, a+b) \subset V(\mathcal{A}, a)+V(\mathcal{A}, b)$.

3) $V(\mathcal{A}, \lambda 1+\mu a)=\lambda+\mu V(\mathcal{A}, a)$.

4) $V(\mathcal{A}, a)=V(\mathcal{A}, a, 1)$.

5) $\sigma(a) \subset V(\mathcal{A}, a)$.

The fundamental link between the numerical range of $a \in \mathcal{A}$ and the group $\left\{e^{t a}: t \in \mathbb{R}\right\}$ is as follows:

$$
\begin{aligned}
& \max \{\operatorname{Re} \lambda: \lambda \in V(\mathcal{A}, a)\}=\sup \left\{\frac{1}{t} \log \left\|e^{t a}\right\|: t>0\right\} \\
& =\lim _{t \rightarrow 0^{+}} \frac{1}{t} \log \left\|e^{t a}\right\|=\inf \left\{\frac{1}{t}(\|1+t a\|-1): t>0\right\} \\
& =\lim _{t \rightarrow 0^{+}}\left\{\frac{1}{t}(\|1+t a\|-1)\right\}
\end{aligned}
$$

By Lemma 5.2 of [6], an element $a \in \mathcal{A}$ is said to be hermitian if $V(\mathcal{A}, a) \subset \mathbb{R}$ or equivalently $\left\|e^{\text {ta }}\right\|=1$ $(t \in \mathbb{R})$, equivalently $\lim _{t \rightarrow 0^{+}} \frac{1}{t}(\|1+t a\|-1)=0$.

We denote the set of all hermitian elements of $\mathcal{A}$ by $\mathcal{H}(\mathcal{A})$. It is well-known that if $h \in \mathcal{H}(\mathcal{A})$ then the convex hull of the spectrum satisfies

$\operatorname{co}(\sigma(h))=V(\mathcal{A}, h)$ and $r(h)=\|h\|$. Also $\mathcal{H}(\mathcal{A})$ is closed real subspace of $\mathcal{A}$ and $\mathcal{H}(\mathcal{A}) \cap i \mathcal{H}(\mathcal{A})=\{0\}$ and if $h, k \in \mathcal{H}(\mathcal{A})$ then $i(h k-k h) \in \mathcal{H}(\mathcal{A})$

Furthermore, if $\mathcal{A}$ is a $B^{*}$-algebra, then by Example 5.3 of [6], $a \in \mathcal{H}(\mathcal{A})$ if and only if $a^{*}=a$. 
An element $a \in \mathcal{A}$ is called positive if

$V(\mathcal{A}, a) \subset \mathbb{R}^{+}$. We denote the set of all positive elements of $\mathcal{A}$ by $\mathcal{A}^{+}$. By Theorem 5.14 of [6], $a \in \mathcal{A}^{+}$ if and only if $a \in \mathcal{H}(\mathcal{A})$ and $\sigma(a) \subseteq[0, \infty)$. In the real Banach space $\mathcal{H}(\mathcal{A})$, the set $\mathcal{A}^{+}$is a normal closed cone in which $\mathbf{1}$ is an interior point. Let

$\mathcal{J}(\mathcal{A})=\mathcal{H}(\mathcal{A})+i \mathcal{H}(\mathcal{A})$. Since $\mathcal{H}(\mathcal{A}) \cap \mathcal{H}(\mathcal{A})=\{0\}$, hence each element of $\mathcal{J}(\mathcal{A})$ has a unique representation of the form $h+i k$ with $h, k \mathcal{H}(\mathcal{A})$. If we define a linear involution $*$ from $\mathcal{J}(\mathcal{A})$ to itself by

$(h+i k)^{*}=h-i k$ then $\mathcal{J}(\mathcal{A})$ with the norm of $a \in \mathcal{A}$ is a complex Banach space and $*$ is a continuous linear involution on $\mathcal{J}(\mathcal{A})$. In the general case $\mathcal{J}(\mathcal{A})$ is not an algebra and $*: \mathcal{J}(\mathcal{A}) \rightarrow \mathcal{J}(\mathcal{A})$ is not an involution because in particular $(a b)^{*} \neq b^{*} a^{*} \quad(a, b \in \mathcal{J}(\mathcal{A}))$. However, if $\mathcal{A}=\mathcal{J}(\mathcal{A})$ and for every $u \in \mathcal{H}(\mathcal{A})$, $u^{2}=h+i k$ with $h k=k h, h, k \mathcal{H}(\mathcal{A})$, then $\mathcal{A}$ is a complex unital $C^{*}$-algebra with continuous involution * and $\mathcal{H}(\mathcal{A})$ is its set of self-adjoint elements [6].

We say that $u \in \mathcal{A}$ is normal if $u=h+i k$ with $h, k \in \mathcal{H}(\mathcal{A})$ and $h k=k h$. Observe that $u \in \mathcal{A}$ is normal if and only if $u \in \mathcal{J}(\mathcal{A})$ and $u u^{*}=u^{*} u$. An element $u \in \mathcal{J}(\mathcal{A})$ satisfying $u=u u^{*} u$ is called a partial isometry.

Definition 1.2. Let $\mathcal{A}$ be a complex unital $C^{*}$-algebra, then $a \in \mathcal{A}$ is called an orthogonal projection if $a^{2}=a=a^{*}$. Moreover $a \in \mathcal{A}$ is called a Moore-Penrose invertible if there exists some $b \in \mathcal{A}$ such that

$$
a b a=a, b a b=b,(a b)^{*}=a b,(b a)^{*}=b a .
$$

In this case $b$ is the Moore-Penrose inverse of $a$ and usually denoted by $a^{\dagger}$. If $a$ is Moore-Penrose invertible, then $a^{\dagger}$ is unique.

Definition 1.3. Let $\mathcal{A}$ be a complex unital Banach algebra. An element $a \in \mathcal{A}$ is called an orthogonal projection if $a^{2}=a$ and $a \in \mathcal{H}(\mathcal{A})$. Moreover $a \in \mathcal{A}$ is called a Moore-Penrose invertible if there exists $b \in \mathcal{A}$ such that

$$
a b a=a \quad \& \quad b a b=b \forall a b, a b \in \mathcal{H}(\mathcal{A}),
$$

then the element $b$ is called the Moore-Penrose inverse of $a$, and it also will be denoted by $a^{\dagger}$. The MoorePenrose inverse of $a$ is unique in the case when it exists.

If $a$ is Moore-Penrose invertible then the equality $a a^{\dagger}=a^{\dagger} a$ does not hold in general. Hence it is interesting to distinguish such elements.

Definition 1.4. An element $a$ of a unital Banach algebra $\mathcal{A}$ is said to be $e$-projection if there exists $a^{\dagger}$ and $a a^{\dagger}=a^{\dagger} a$.

If $S \subseteq \mathcal{A}$, we define the centralizer of $S$ by

$$
\Gamma(S)=\{\mathfrak{a} \in \mathcal{A}: \mathfrak{a} s=s \mathfrak{a} \forall s \in S\} .
$$

We say that $S$ commutes if any two elements of $S$ commute with each other. If $S \subseteq \mathcal{A}$ is commutes and $\mathcal{B}=\Gamma(\Gamma(S))$ then by Theorem 11.22 of [7] $\mathcal{B}$ is a commutative Banach algebra (with unit 1), $S \subseteq \mathcal{B}$ and $\sigma_{\mathcal{A}}(a)=\sigma_{\mathcal{B}}(a)$ for every $a \in \mathcal{B}$.

Lemma 1.5. [7]. Let $\mathcal{A}$ be a complex unital Banach algebra, let $u=h+i k \in \mathcal{J}(\mathcal{A})$ be a normal element, $\mathcal{B}(u)=\Gamma(\Gamma(\{h, k\}))$. If $\Delta_{u}$ is the set of all nontrivial complex homomorphisms of $\mathcal{B}(u)$. Then

1) $\sigma(b)=\left\{\tau(b): \tau \in \Delta_{u}\right\}$.

2) $\tau(h), \tau(k) \in \mathbb{R} \forall \tau \in \Delta_{u}$

3) $\tau\left(u^{*}\right)=\overline{\tau(u)}$ for all $\tau \in \Delta_{u}$ and $\sigma\left(u^{*}\right)=\overline{\sigma(u)}$

4) If $a, b \in \mathcal{B}(u)$, then

$$
\sigma(a+b) \subseteq \sigma(a)+\sigma(b), \sigma(a b) \subseteq \sigma(a) \sigma(b)
$$

\section{2. g-Orthogonal Projections and Generalized e-Projections}

Definition 2.1. An element $u \in \mathcal{J}(\mathcal{A})$ is called generalized orthogonal projection or simply a $g$-orthogonal projection if there exists a natural number $n \geq 2$ such that $u^{n}=u^{*}$ Also $u$ is said to be generalized e-projection if there exists $u^{\dagger}$ and $u^{n}=u^{\dagger}$

Theorem 2.2. Suppose that $u \in \mathcal{J}(\mathcal{A})$ is a $g$-orthogonal projection. Then

1) $u$ is normal.

2) $\sigma(u) \subseteq\{0\} \cup\left\{e^{i \frac{2 k \pi}{n+1}}: 0 \leq k \leq n\right\}$.

3) If $h^{n-2 j+1} k^{2 j-1} \in \mathcal{H}(\mathcal{A})$ for all $1 \leq j \leq\left\lfloor\frac{n}{2}\right\rfloor$ then $\left(u^{*}\right)^{n}=u$ and $u^{n^{2}}=u$

Proof.

1) Since $u^{n}=u^{*}$ hence we have

$$
u u^{*}=u^{n+1}=u^{*} u .
$$

2) Let $\lambda \in \sigma(u)$, then by the Lemma 1.5 there is a $\tau \in \Delta_{u}$ such that $\lambda=\tau(u)$, thus we have

$$
\bar{\lambda}=\tau\left(u^{*}\right)=\tau\left(u^{n}\right)=\tau(u)^{n}=\lambda^{n} .
$$

Now if $\lambda \neq 0$, then $|\lambda|=1$ and hence $\lambda^{n+1}=1$ which implies that $\lambda=e^{i \frac{2 k \pi}{n+1}}$ with $0 \leq k \leq n$

3) Since $u^{n}=u^{*}$ hence

$$
\begin{aligned}
& -h+\sum_{j=0}^{\left\lfloor\frac{n}{2}\right\rfloor}(-1)^{j}\left(\begin{array}{c}
n \\
2 j
\end{array}\right) h^{n-2 j} k^{2 j} \\
& =i\left(-k-\sum_{j=1}^{\left.\frac{n}{2}\right\rfloor+1}(-1)^{j-1}\left(\begin{array}{c}
n \\
2 j-1
\end{array}\right) h^{n-2 j+1} k^{2 j-1}\right) .
\end{aligned}
$$

Using the Lemma 1.6 (4) we have 


$$
\sigma\left(-h+\sum_{j=0}^{\left\lfloor\frac{n}{2}\right\rfloor}(-1)^{j}\left(\begin{array}{c}
n \\
2 j
\end{array}\right) h^{n-2 j} k^{2 j}\right) \subseteq \mathbb{R}
$$

and

$$
\sigma\left(-k-\sum_{j=1}^{\left\lfloor\frac{n}{2} \mid+1\right.}(-1)^{j-1}\left(\begin{array}{c}
n \\
2 j-1
\end{array}\right) h^{n-2 j+1} k^{2 j-1}\right) \subseteq \mathbb{R} .
$$

This yields

$$
\begin{aligned}
& \sigma\left(-k-\sum_{j=1}^{\left.\frac{n}{2}\right\rfloor+1}(-1)^{j-1}\left(\begin{array}{c}
n \\
2 j-1
\end{array}\right) h^{n-2 j+1} k^{2 j-1}\right) \\
& \subseteq \mathbb{R} \cap i \mathbb{R}=\{0\} .
\end{aligned}
$$

Thus we have

$$
r\left(-k-\sum_{j=1}^{\left\lfloor\frac{n}{2}\right\rfloor+1}(-1)^{j-1}\left(\begin{array}{c}
n \\
2 j-1
\end{array}\right) h^{n-2 j+1} k^{2 j-1}\right)=0 .
$$

Since

$$
-k-\sum_{j=1}^{\left\lfloor\frac{n}{2}\right\rfloor+1}(-1)^{j-1}\left(\begin{array}{c}
n \\
2 j-1
\end{array}\right) h^{n-2 j+1} k^{2 j-1} \in \mathcal{H}(\mathcal{A}),
$$

hence

$$
\left\|-k-\sum_{j=1}^{\left\lfloor\frac{n}{2} \mid+1\right.}(-1)^{j-1}\left(\begin{array}{c}
n \\
2 j-1
\end{array}\right) h^{n-2 j+1} k^{2 j-1}\right\|=0 .
$$

which shows that

$$
\begin{gathered}
h=\sum_{j=0}^{\left\lfloor\frac{n}{2}\right\rfloor}(-1)^{j}\left(\begin{array}{c}
n \\
2 j
\end{array}\right) h^{n-2 j} k^{2 j}, \\
-k=\sum_{j=1}^{\left\lfloor\frac{n}{2}\right\rfloor+1}(-1)^{j-1}\left(\begin{array}{c}
n \\
2 j-1
\end{array}\right) h^{n-2 j+1} k^{2 j-1} .
\end{gathered}
$$

Therefore

$$
\begin{gathered}
\left(u^{*}\right)^{n}=\sum_{j=0}^{\left\lfloor\frac{n}{2}\right\rfloor}(-1)^{j}\left(\begin{array}{c}
n \\
2 j
\end{array}\right) h^{n-2 j} k^{2 j} \\
-i \sum_{j=1}^{\left\lfloor\frac{n}{2}\right\rfloor+1}(-1)^{j-1}\left(\begin{array}{c}
n \\
2 j-1
\end{array}\right) h^{n-2 j+1} k^{2 j-1}=h-i(-k)=u
\end{gathered}
$$

The second implication is obvious.

Theorem 2.3. Let $u \in \mathcal{J}(\mathcal{A})$ be a generalized e-projection. Then

1) $u^{n+2}=u$ and $u$ is an $e$-projection.

2) $\sigma(u) \subseteq\{0\} \cup\left\{e^{i \frac{2 k \pi}{n+1}}: 0 \leq k \leq n\right\}$.

\section{Proof.}

1) Since $u^{n}=u^{\dagger}$ hence we have $u=u u^{\dagger} u=u^{n+2}$ and $u u^{\dagger}=u^{n+1}=u^{\dagger} u$.

2) This follows immediately from Theorem 2.2.
Theorem 2.4. Let $u \in \mathcal{J}(\mathcal{A})$ and

$$
\sigma(u) \subseteq\{0\} \cup\left\{e^{i \frac{2 k \pi}{n+1}}: 0 \leq k \leq n\right\} .
$$

Then the following statements hold:

1) If $u$ is normal, then $r\left(u^{n}-u^{*}\right)=0$.

2) If $h k,\left(h^{2}\right.$ ork $\left.^{2}\right), h^{n-j} k^{j} \in \mathcal{H}(\mathcal{A})$ for all $1 \leq j \leq n$, then $u$ is a $g$-orthogonal projection.

\section{Proof.}

1) Put $v=u^{n}-u^{*}$ Since $u$ is normal hence $h k=k h$ and so $v \in \mathcal{B}(u)$ Now suppose that $\lambda \in \sigma(v)$, then there exists some $\tau \in \Delta_{u}$ with $\lambda=\tau(v)=\tau(u)^{n}-\overline{\tau(u)}$. Let $\tau(u) \neq 0$, since $\tau(u) \in \sigma(u)$ thus $\tau(u)^{n+1}=|\tau(u)|=1$. This shows that $\lambda \tau(u)=\tau(u)^{n+1}-|\tau(u)|^{2}=0$, and so $\sigma(v)=\{0\}$ From this we have $r\left(u^{n}-u^{*}\right)=0$.

2) By the Murphy's Theorem [8], $h k=k h$ so $u$ is normal. Now from $v=u^{n}-u^{*}$ we obtain

$$
\begin{aligned}
& v+h-\sum_{j=0}^{\left\lfloor\frac{n}{2}\right\rfloor}(-1)^{j}\left(\begin{array}{c}
n \\
2 j
\end{array}\right) h^{n-2 j} k^{2 j} \\
& =i\left(k+\sum_{j=1}^{\frac{n}{2} \mid+1}(-1)^{j-1}\left(\begin{array}{c}
n \\
2 j-1
\end{array}\right) h^{n-2 j+1} k^{2 j-1}\right)
\end{aligned}
$$

Using the Lemma 1.6(4) and applying (1) we have

$$
\begin{aligned}
& \sigma\left(k+\sum_{j=1}^{\left\lfloor\frac{n}{2}\right\rfloor+1}(-1)^{j-1}\left(\begin{array}{c}
n \\
2 j-1
\end{array}\right) h^{n-2 j+1} k^{2 j-1}\right) \\
& \subseteq \mathbb{R} \cap i \mathbb{R}=\{0\} .
\end{aligned}
$$

Since $h^{n-j} k^{j} \in \mathcal{H}(\mathcal{A})$ for all $1 \leq j \leq n$, hence

$$
k+\sum_{j=1}^{\left\lfloor\frac{n}{2}\right\rfloor+1}(-1)^{j-1}\left(\begin{array}{c}
n \\
2 j-1
\end{array}\right) h^{n-2 j+1} k^{2 j-1}=0 .
$$

This shows that

$$
v=\sum_{j=0}^{\left\lfloor\frac{n}{2}\right\rfloor}(-1)^{j}\left(\begin{array}{c}
n \\
2 j
\end{array}\right) h^{n-2 j} k^{2 j}-h \in \mathcal{H}(\mathcal{A}) .
$$

Since $r(v)=0$, thus $v=0$, which implies that $u^{n}=u^{*}$.

Theorem 2.5. An element $u \in \mathcal{A}$ is a $g$-orthogonal projection if and only if $u$ is normal and

$$
\sigma(u) \subseteq\{0\} \cup\left\{e^{i \frac{2 k \pi}{n+1}}: 0 \leq k \leq n\right\} .
$$

Proof. If $u$ is a $g$-orthogonal projection then the implication follows from the Theorem 2.2. Conversely suppose that $u$ is normal and 


$$
\sigma(u) \subseteq\{0\} \cup\left\{e^{i \frac{2 k \pi}{n+1}}: 0 \leq k \leq n\right\} .
$$

For every $\lambda \in \sigma(u)$ we define the Reiesz projection of $u$ associated with $\lambda$ by $E(\lambda)=\frac{1}{2 \pi i} \int_{C_{\lambda}}(\alpha 1-u)^{-1} \mathrm{~d} \alpha$ where $C_{\lambda}$ is a smooth closed curve which $\lambda$ interior to $C_{\lambda}$ and $\sigma(u) \backslash\{\lambda\}$ exterior to $C_{\lambda}$. Then by Proposition VII.4.11 of [9], $u$ has the representation as folslows:

$$
u=0 E(0) \oplus \sum_{k=0}^{n} \oplus e^{i \frac{2 k \pi}{n+1}} E\left(e^{i \frac{2 k \pi}{n+1}}\right)
$$

where $E(\lambda)=0$ for all

$$
\lambda \in\left\{0, e^{i \frac{i k \pi}{n+1}}: 0 \leq k \leq n\right\}-\sigma(u)
$$

and $E(\lambda)^{2}=E(\lambda)$ and

$$
\sum_{\lambda \in \sigma(u)} \oplus E(\lambda)=I d_{\mathcal{A}}
$$

and $E(\lambda) E(\mu)=E(\mu) E(\lambda)$ for $\lambda, \mu \in \sigma(u)$ and $\lambda \neq \mu$. Now we compute

$$
\begin{aligned}
u^{n} & =\left(0 E(0) \oplus \sum_{k=0}^{n} \oplus e^{i \frac{2 k \pi}{n+1}} E\left(e^{i \frac{2 k \pi}{n+1}}\right)\right)^{n} \\
& =0 E(0) \oplus \sum_{k=0}^{n} \oplus e^{i \frac{2 k n \pi}{n+1}} E\left(e^{i \frac{2 k \pi}{n+1}}\right) \\
& =0 E(0) \oplus \sum_{k=0}^{n} \oplus e^{-i \frac{2 k \pi}{n+1}} E\left(e^{i \frac{2 k \pi}{n+1}}\right)=u^{*}
\end{aligned}
$$

Theorem 2.6. Suppose that $u \in \mathcal{J}(\mathcal{A})$ and

$$
\sigma(u) \subseteq\{0\} \cup\left\{e^{i \frac{2 k \pi}{n+1}}: 0 \leq k \leq n\right\} .
$$

If $u$ has the representation

$$
u=\sum_{\lambda \in \sigma(u) \backslash\{0\}} \oplus \lambda E(\lambda),
$$

where

$$
E(\lambda)=\frac{1}{2 \pi i} \int_{C_{\lambda}}(\alpha 1-u)^{-1} \mathrm{~d} \alpha
$$

is a Riesz projection of $u$ associated with $\lambda$ and $C_{\lambda}$ is a smooth closed curve which $\lambda$ interior to $C_{\lambda}$ and $\sigma(u) \backslash\{0\}$ exterior to $C_{\lambda}$. Then $u$ is a generalized $e$ projection.

Proof. Since for all $\lambda \in \sigma(u)$ we have $\lambda^{n}=\lambda^{-1}$ hence

$$
\begin{aligned}
u^{n} & =\left(\sum_{\lambda \in \sigma(u)} \oplus \lambda E(\lambda)\right)^{n} \\
& =\left(0 E(0) \oplus \sum_{\lambda \in \sigma(u) \backslash\{0\}} \oplus \lambda E(\lambda)\right)^{n} \\
& =0 E(0) \oplus \sum_{\lambda \in \sigma(u) \backslash\{0\}} \oplus \lambda^{n} E(\lambda) \\
& =0 E(0) \oplus \sum_{\lambda \in \sigma(u)\{0\}} \oplus \lambda^{-1} E(\lambda) \\
& =0 E(0) \oplus\left(\sum_{\lambda \in \sigma(u) \backslash 0\}} \oplus \lambda E(\lambda)\right)^{-1}=u^{\dagger}
\end{aligned}
$$

In the general case if $h \in \mathcal{H}(\mathcal{A})$ then it does not follows that $h^{2} \notin \mathcal{H}(\mathcal{A})$.

Example 2.7. Let $\mathcal{A}=\mathbb{C}^{3}$ with pointwise multiplication and let $p: \mathcal{A} \rightarrow[0, \infty)$ be defined by

$$
p(\alpha, \beta, \gamma)=\sup _{\substack{\lambda \in \mathbb{C} \\|\lambda|=1}}|\bar{\lambda} \alpha+\beta+\lambda \gamma| .
$$

Define the norm $\|\cdot\|$ on $\mathcal{A}$ by

$$
\|a\|=\sup _{\substack{x \in \mathcal{A} \\ p(x)=1}} p(x a) \forall a \in \mathcal{A} .
$$

Then $(\mathcal{A},\|\cdot\|)$ is a complex commutative Banach algebra with unit $\mathbf{1}=(1,1,1)$. If $h=(-1,0,1)$ then the following properties are shown in [6].

$$
\begin{gathered}
\mathcal{A}=\left\{\alpha 1+\beta h+\gamma h^{2}: \alpha, \beta, \gamma \in \mathbb{C}\right\}, \\
\mathcal{H}(\mathcal{A})=\{\alpha 1+\beta h: \alpha, \beta \in \mathbb{R}\}, \\
\mathcal{J}(\mathcal{A})=\{\xi 1+\eta h: \xi, \eta \in \mathbb{C}\},
\end{gathered}
$$

and $h \in \mathcal{H}(\mathcal{A})$ but $h^{2} \notin \mathcal{H}(\mathcal{A})$ and each element of $\mathcal{J}(\mathcal{A})$ is normal. Furthermore if $u=\xi 1+\eta h \in \mathcal{J}(\mathcal{A})$, then $u^{*}=\bar{\xi} 1+\bar{\eta} h$.

Lemma 2.8. Let $\mathcal{A}$ as in Example 2.7. Then $u \in \mathcal{H}(\mathcal{A})$ is a $g$-orthogonal projection if and only if

$$
\sigma(u) \subseteq\{0\} \cup\left\{e^{i \frac{2 k \pi}{n+1}}: 0 \leq k \leq n\right\} .
$$

Proof. For all $(\alpha, \beta, \gamma) \in \mathcal{A}$ we have

$$
\sigma(\alpha, \beta, \gamma)=\{\alpha, \beta, \gamma\}
$$

hence $\sigma(v)=\{0\}$ if and only if $v=0$. Now if

$$
\sigma(u) \subseteq\{0\} \cup\left\{e^{i \frac{2 k \pi}{n+1}}: 0 \leq k \leq n\right\},
$$

then by Theorem 2.4, we have $\sigma\left(u^{n}-u^{*}\right)=\{0\}$ which implies that $u^{n}=u^{*}$. The converse implication follows immediately from Theorem 2.2.

Lemma 2.9. Let $\mathcal{A}$ as in Example 2.7 and $u \in \mathcal{J}(\mathcal{A})$. Then the following conditions are equivalent:

1) $\sigma(u) \subseteq\{0\} \cup\left\{e^{i \frac{k \pi}{2}}: 0 \leq k \leq 3\right\}$.

2) $u^{3}=u^{*}$. 
3) $u \in\left\{(\lambda, \lambda, \lambda),(-\lambda, 0, \lambda): \lambda=0 \vee \lambda^{4}=1\right\}$.

4) $u^{5}=u$.

\section{Proof.}

1) $\Rightarrow$ 2) follows from the Lemma 2.8.

2) $\Rightarrow$ 3) Let $u=\xi 1+\eta h \in \mathcal{J}(\mathcal{A})$ Since $u^{3}=u^{*}$ hence

$$
\left((\xi-\eta)^{3}, \xi^{3},(\xi+\eta)^{3}\right)=(\bar{\xi}-\bar{\eta}, \bar{\xi}, \bar{\xi}+\bar{\eta}) .
$$

If $\xi=0$ then $\eta^{3}=\bar{\xi}$, thus $u=(-\eta, 0, \underline{\eta})$ with $\eta=0$ or $\eta^{4}=1$. Let $\xi \neq 0$ Since $\xi^{3}=\bar{\xi}$ hence $-3 \xi^{2} \eta+3 \xi \eta^{2}-\eta^{3}=-\bar{\eta}$ and $3 \xi^{2} \eta+3 \xi \eta^{2}+\eta^{3}=\bar{\eta}$. It follows $\xi \eta^{2}=0$, therefore $\eta=0$. This shows that $u=(\xi, \xi, \xi)$ with $\xi^{4}=1$.

(3) $\Rightarrow$ (4): Clear.

(4) $\Rightarrow$ (1): follows from the spectral mapping theorem.

Theorem 2.10. Let $u, v \in \mathcal{J}(\mathcal{A})$ be $g$-orthogonal projections such that $u v=v u=0$. Then $u+v$ is a $g$-orthogonal projection.

Proof. By the hypotheses

$$
v^{j} u^{k}=u^{j} v^{k}=0
$$

for all $1 \leq j, k \leq n-1$ hence we have

$$
\begin{aligned}
(u+v)^{n}= & u^{n}+v^{n}+\sum_{\substack{i \geq 1, j \geq 1 \\
i+j=n}}\left(u^{i} v^{j}+v^{i} u^{j}\right) \\
& +\sum_{\substack{i \geq 1, j \geq 1, k \geq 1 \\
i+j+k=n}}\left(u^{i} v^{j} u^{k}+v^{i} u^{j} v^{k}\right) \\
& +\cdots+(\underbrace{u v u v \cdots u}_{n \text { time }}+\underbrace{v u v u \cdots v}_{n \text { time }}) \\
= & u^{*}+v^{*}=(u+v)^{*} .
\end{aligned}
$$

\section{Acknowledgements}

The author expresses his gratitude to the referee for carefully reading of the manuscript and giving useful comments.

\section{REFERENCES}

[1] E. Berkson, "Hermitian Projections and Orthogonality in Banach Spaces," Proceedings London Mathematical Society, Vol. 3, No. 24, 1972, pp. 101-118. doi:10.1112/plms/s3-24.1.101

[2] C. Schmoeger, "Generalized Projections in Banach Algebras," Linear Algebra and Its Applications, Vol. 430, No. 10, 2009, pp. 601-608. doi:10.1016/j.laa.2008.07.020

[3] H. Du and Y. Li, "The Spectral Characterization of Generalized Projections," Linear Algebra and its Applications, Vol. 400, 2005, pp. 313-318. doi:10.1016/j.laa.2004.11.027

[4] I. Groß and G. Trenkler, "Generalized and Hyper Generalized Projectors," Linear Algebra and its Applications, Vol. 264, 1997, pp. 463-474.

[5] L. Lebtahi and N. thome, "A Note on $\kappa$-Generalized Projection," Linear Algebra and Its Applications, Vol. 420, 2007, pp. 572-575. doi:10.1016/j.laa.2006.08.011

[6] F. F. Bonsal and J. Duncan, "Numerical Ranges of Operators on Normed Spaces and Elements of Normed Algebras,” Cambridge University Press, Cambridge, 1971.

[7] W. Rudin, "A Course in Functional Analysis," McGraw Hill, New York, 1973.

[8] I. S. Murphy, "A Note on Hermitian elements of a Banach Algebra," Journal London Mathematical Society, Vol. 3, No. 6, 1973, pp. 427-428. doi: $10.1112 / \mathrm{jlms} / \mathrm{s} 2-6.3 .427$

[9] J. B. Conway, "A Course in Functional Analysis," Springer-Verlag Inc., New York, 1985. 